\title{
Bicolor Electroluminescent Pixels from Single Active Molecular Material
}

\author{
Ilenia Viola $,{ }^{*},{ }^{\dagger}$ Claudia Piliego,${ }^{\dagger}$ Laura Favaretto, $^{\dagger}$ Giovanna Barbarella, ${ }^{\mp}$ \\ Roberto Cingolani, ${ }^{\dagger, \S}$ and Giuseppe Gigli ${ }^{*}, \dagger$
}

National Nanotechnology Laboratory (NNL) of INFM-CNR, Distretto tecnologico ISUFI, Via Arnesano, 73100 Lecce, Italy, Istituto Italiano di Tecnologia (IIT), Via Morego, 30, 16163 Genova, Italy, Istituto per la Sintesi Organica e la Fotoreattività (ISOF), Area di Ricerca CNR, Via Gobetti, 101, 40129 Bologna, Italy

\begin{abstract}
We report on the fabrication of the first bicolor micropixelated OLED from a single molecular material using a singlestep bottom up procedure. The implementation of a deposition technique, based on a spatial-switch and conformational-sensitive STD surface-tension-driven lithography, has allowed us to exploit the spontaneous supramolecular properties and the conformational flexibility of a conjugated thiophene-based material, 6-bis-(50-hexyl-[2, 20]bithiophen-5-yl)-3, 5-dimethyl-dithieno[3, 2-b; 20, 30-d]thiophene (DTT7Me). The existence of two regularly alternating emitting regions on a micrometer scale allows obtaining electroluminescent emission at two different wavelengths from a single material.
\end{abstract}

KEYWORDS: surface tension-driven deposition $\bullet$ wettability $\bullet$ oligothiophene $\bullet$ OLED

\section{INTRODUCTION}

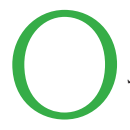
rganic light-emitting diodes (OLEDs) based on $\pi$-conjugated molecules and polymers have recently showed most relevant characteristics for commercial applications such as large-area, ultrathin, flexible displays and lighting devices (1).

Even if a few products based on organic materials have already appeared on the market, the possibility to exploit low-cost and single-step patterning techniques that allow manipulating unusual chemically and mechanically fragile organic materials on manifold substrates is still a challenge $(2-4)$

These manufacturing techniques aspire to a multiscale control of self-organization of molecular compounds through confinement at the interface, a minimization of the materials amount and processing cost, as well as the exploitation of flexibility and functionality of organic materials (5).

To this aim, unconventional processing technique, based on the spontaneous supramolecular organization and conformational modifications of conjugated functional materials, has not been fully explored (6). $\pi$-conjugated systems are in fact characterized by a direct relationship between the electro-optical properties and the supramolecular structure $(7,8)$. In particular, a fine-tuning of the optical properties can be obtained by controlling the intra- and intermolecular interactions at molecular level through micro and nanopatterning procedures $(8,9)$.

\footnotetext{
* Corresponding author. E-mail: ilenia.viola@unile.it (I.V.); giuseppe.gigli@ unile.it (G.G.).

Received for review October 21, 2009 and accepted January 11, 2010

${ }^{\dagger}$ National Nanotechnology Laboratory (NNL) of INFM-CNR

* Istituto per la Sintesi Organica e la Fotoreattività.

§ Istituto Italiano di Tecnologia.

DOI: 10.1021 /am9007227

๑ 2010 American Chemical Society
}

In this context, thiophene-based materials, distinguished among $\pi$-conjugated systems for their notable chargetransport and light-emission properties, are characterized by a strong relationship between their electro-optical properties and the conformational structure, thus giving the possibility to finely tune, for example, their optical properties in a wide spectral range, from UV to near IR, through extremely varied functionalizations and properly conformation changes $(8,10)$.

In our previous works, we have shown how the control of the thermodynamic phenomena at the interface of liquid thin films in nonwetting conditions allows for the fabrication of pixelated patterns of functional light-emitting materials with well-resolved features. By modulating the solvent polarity, we have succeeded in selectively addressing the instability phenomena of a liquid film to obtain monochromatic OLEDs with positive or negative pixelated structures over large area (11).

In this paper, we report, for the first time, the fabrication of a bicolor micro pixel OLED array realized by a single luminescent molecular material, using a single-step lithographic approach based on a surface-tension-driven (STD) lithography.

\section{EXPERIMENTAL SECTION}

Microstructure Fabrication. Molecular bicolor pixel microarrays were realized by depositing $9 \mu \mathrm{L}$ of a freshly prepared $0.2 \%$ $(\mathrm{w} / \mathrm{v})$ solution of DTT7Me in warm toluene on a copper $(\mathrm{Cu})$ template fixed on different substrates. Details of the synthesis of the oligothiophene derivative (DTT7Me) are reported in refs $11 \mathrm{~b}$ and 12. The ITO-covered glass substrates (Vision Tek Systems LTD) were cleaned in an ultrasonic bath with acetone, 2-propanol, and deionized water (10 min for each step). The slow dewetting process has been carried on at ambient temperature, $T_{\text {slow }}=24^{\circ} \mathrm{C}$, under controlled conditions, whereas the fast process has been realized on a hot plate at $T_{\text {fast }}=80^{\circ} \mathrm{C}$.

Two different kinds of substrates were prepared for the device fabrication. In the first case we performed an $\mathrm{O}_{2}$ plasma 
a)

b)
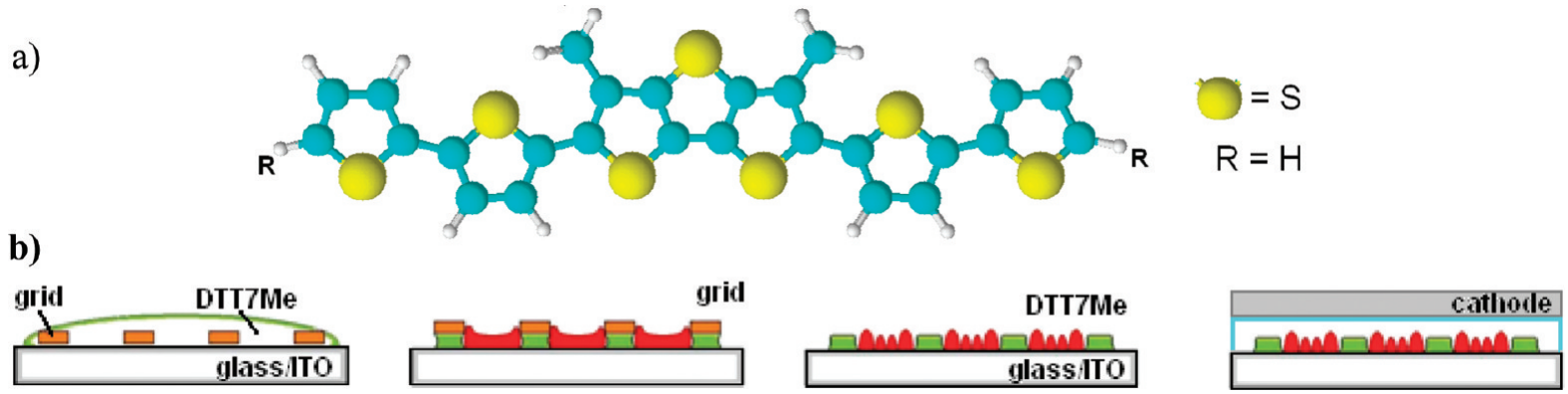

FIGURE 1. (a) Molecular structure of DTT7Me. (b) Sketch of the STD deposition technique used for the realization of the DTT7Me bicolor pixelated OLED. A drop of dilute solutions of DTT7Me is deposited on a template mesh, set on a glass/ITO substrate; during the solvent evaporation, the DTT7Me molecules move both under and inside the mesh; the interplay of the energetic conditions at the different interfaces drives the molecular self-assembly and realizes a bicoloured pixels structure; the OLED is realized on the bicolor pattern.

treatment $(P=50 \mathrm{~W}, t=60 \mathrm{~s})$ to remove any organic residues and then we deposited a PEDOT:PSS layer $(80 \mathrm{~nm})$ by spincoating (4000 rpm for $60 \mathrm{~s}$ ) and we dried it on a hot plate at $180^{\circ}$ for $10 \mathrm{~min}$. In the second case, we evaporated a layer of $4,4^{\prime}-N, N^{\prime}$-dicarbazole-biphenyl (CBP) $(40 \mathrm{~nm})$ at a pressure of 2 $\times 10^{-6}$ mbar on the ITO/ PEDOT:PSS substrates.

The surface energy of all the interfaces were obtained by interpolating the polar and dispersion contributes of the relative contact angles, collected respectively by using water $\left(\mathrm{H}_{2} \mathrm{O}\right)$ and diiodomethane $\left(\mathrm{CH}_{2} \mathrm{I}_{2}\right)$ and detected by the goniometer OCA20Dataphysics.

The morphologies of the active layers on different substrates were characterized by atomic force microscopy (AFM, SolverPro, NT-MDT) in semicontact mode.

Pixel Arrays OLED Fabrication on CBP Substrate. The PEDOT:PSS layer was deposited on the cleaned ITO substrates in order to improve the hole injection and to smooth the ITO surface. Then a CBP (40 nm) layer was used as base level for the deposition of the DTT7Me bicolor pixel microarrays. On the organic patterned layer $40 \mathrm{~nm}$ of 2,9-dimethyl-4,7-diphenyl1,10-phenanthroline (BCP) were deposited in order to flatten the patterned structure. BCP has also the function of hole blocking layer to confine the recombination in the active medium. For the cathode $0.5 \mathrm{~nm}$ of Lithium Fluoride (LiF) and $150 \mathrm{~nm}$ of Aluminum (Al) as capping layer were used. All the layers were evaporated at a pressure of $1 \times 10^{-6} \mathrm{mbar}(1 \mathrm{mbar}$ $=102 \mathrm{~Pa}$ ). The OLED array was biased with a direct current voltage of $10 \mathrm{~V}$. The bicolour pixel array showed a luminance of $75 \mathrm{~cd} / \mathrm{m}^{2}$ and color coordinate $(0.50 ; 0.46)$ in the orangegreen region.

Characterization. The photoluminescence (PL) spectra of the DTT7Me active layer, the $x y$-spatial analysis and the 3D PLreconstructions were obtained by confocal laser scanning microscopy (FluoView1000, Olympus). The electroluminescence (EL) spectra of the OLED pixel arrays were collected by a chargecoupled device (CCD) color camera coupled to the confocal microscope.

\section{RESULTS AND DISCUSSION}

STD lithography of an oligothiophene derivative, 2,6-bis(5'-hexyl-[2,2']bithiophene-5-yl)-3,5-dime thyl- dithieno[3,2.b;2', $3^{\prime}$-d] thiophene (DTT7Me in Figure 1a), on nonwetting substrates suitable for light-emitting devices is reported. The single-step STD deposition procedure, reported in our previous work, has been opportunely modified and optimized in order to obtain two regularly alternating bicolor regions, arranged as a micropixels array over a large area. The pattern generation during the dewetting process is obtained by exploiting the modulation of the energetic parameters at the interfaces and by monitoring the thermodynamic conditions. This allows us to control the conformation and the supramolecular arrangement of the functional material (13). The basic steps of the technique, based on the outward movement of a molecular material dispersed in a solution during the solvent evaporation (usually known as coffee-ring effect) (14), are illustrated in Figure $1 \mathrm{~b}$.

Taking advantage of the structural flexibility of this kind of compounds and the intrinsic capability to modify their inter-ring torsional angles, it is possible to induce changes in their molecular conformation, thus determining different optical properties (15). In our previous work, we demonstrated in particular that DTT7Me assumes, under specific energetic conditions, both a planar and a distorted conformation, characterized by red and green light emission, respectively. More importantly, we have seen that the conformational switch is operated by energetic parameters, regularly alternating at the interface (11b).

Here, the spatial switch of the optical properties has been fully exploited. The understanding of the driving forces of the process allow us to control the different parameters and to realize a bicolor pixelated OLED on different substrates. For this purpose, we first characterized the surface energies at the interface between the solvent and the substrates used (see Table 1), in order to operate a selective control of the molecular dynamics during the self-assembly process in

Table 1. Contact Angles (CAs) and Surface Tension $(\gamma)$ of the Substrates Used for the Pixelated OLED:ITO/ PEDOT:PSS and ITO/CBP

\begin{tabular}{|c|c|c|c|c|c|}
\hline & $\mathrm{CA}\left(\mathrm{H}_{2} \mathrm{O}\right)(\mathrm{deg})$ & $\mathrm{CA}\left(\mathrm{CH}_{2} \mathrm{I}_{2}\right)(\mathrm{deg})$ & $\gamma\left(\mathrm{mN} \mathrm{m}^{-1}\right)$ & $\gamma_{\mathrm{P}}^{a}\left(\mathrm{mN} \mathrm{m}^{-1}\right)$ & $\gamma_{\mathrm{D}}^{b}\left(\mathrm{mN} \mathrm{m}^{-1}\right)$ \\
\hline ITO & $50.0 \pm 0.5$ & $36.0 \pm 0.6$ & 58.85 & 17.29 & 41.56 \\
\hline ITO/ PEDOT-PSS/CBP & $85.3 \pm 0.4$ & $38.0 \pm 0.3$ & 42.42 & 1.82 & 40.60 \\
\hline
\end{tabular}

${ }^{a} \gamma_{\mathrm{P}}$ is the polar contribution to the surface tension. ${ }^{b} \gamma_{\mathrm{D}}$ is the dispersive or apolar contribution to the surface tension, associated wth the van der Waals interactions. 

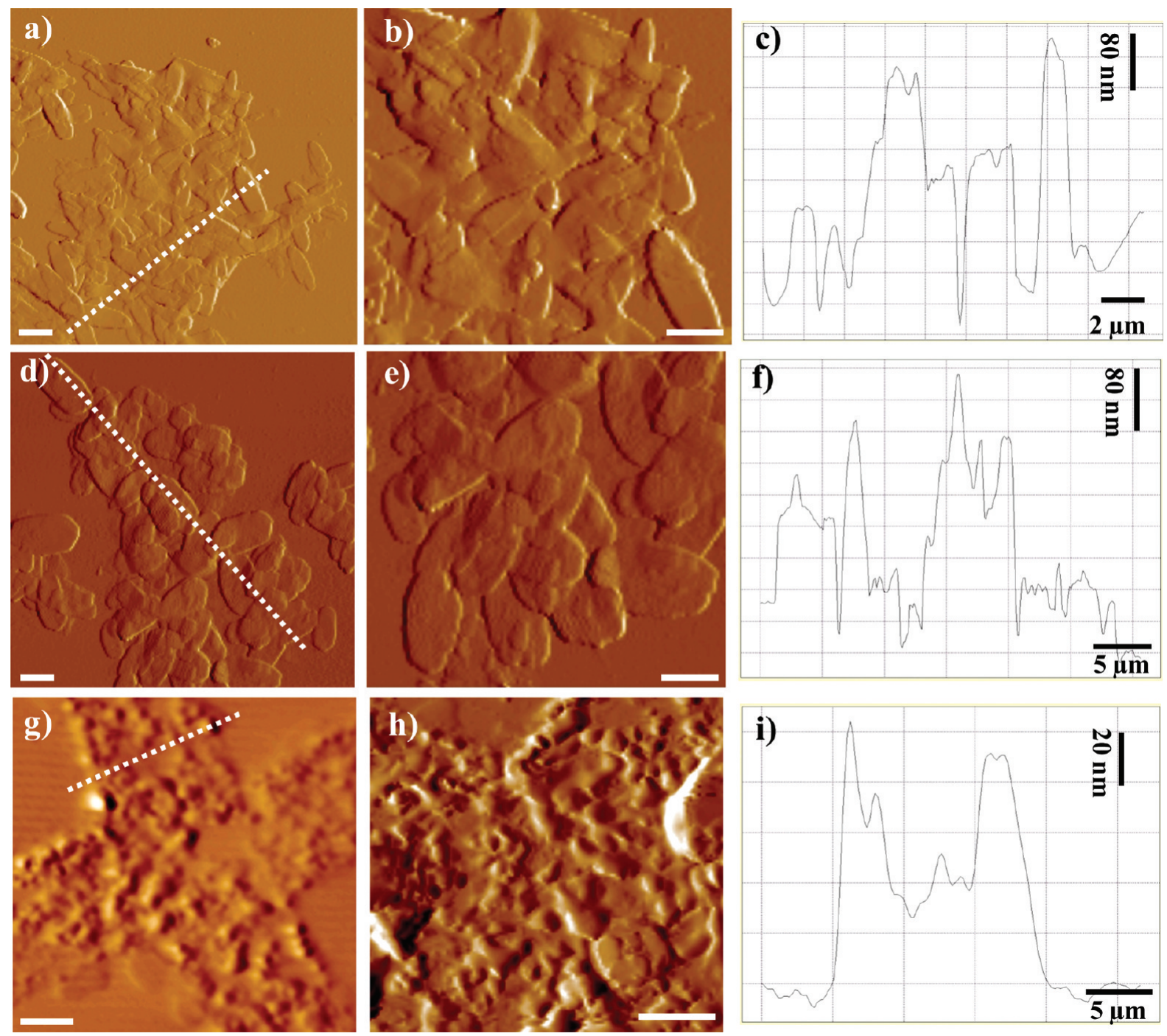

FIGURE 2. $(a-c)$ AFM phase image (a), higher-magnification (b), and height profile (c) of DTT7Me solution deposited on a ITO/PEDOT-PSS substrate; the images represent the molecular aggregates inside the grid. $(d-f)$ DTT7Me molecules self-assembled inside the grid hole on a ITO/PEDOT-PSS/CBP substrate: AFM phase image (d), higher-magnification (e) and height profile (f). (g-i) Self-assembled structure of the molecular material on ITO/ PEDOT-PSS /CBP under the grid mesh. AFM phase image (g), higher-magnification (h), and height profile (i). Scale bars of AFM phase images: $2 \mu \mathrm{m}(\mathrm{a}, \mathrm{b}, \mathrm{d}, \mathrm{e}) ; 5 \mu \mathrm{m}(\mathrm{g}, \mathrm{h})$.
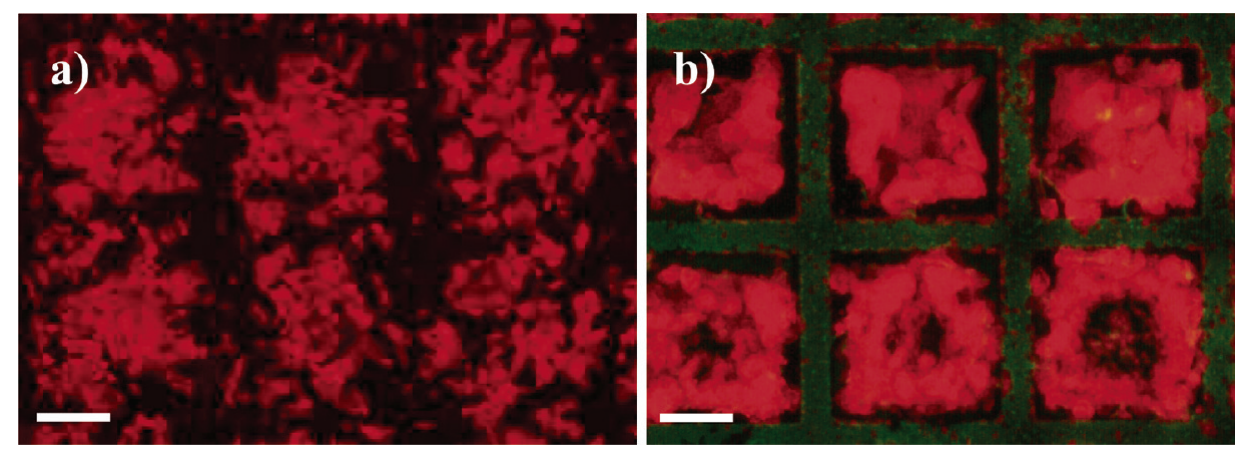

FIGURE 3. Optical image in true color of a DTT7Me film deposited by the STD technique on a CBP substrate (sample B) under (a) slow ( $T_{\text {slow) }}$ and (b) fast $\left(T_{\text {fast }}\right)$ conditions. Red-light emitting crystalline phase is inside the grid hole in both samples, whereas the green amorphous phase is present under the geometrical confinement only by using fast dewetting conditions (b).The scale bar in the images corresponds to $10 \mu \mathrm{m}$.

dewetting conditions. It is well-known, in fact, that local variations of the energetic conditions affect the spontaneous aggregation of molecular materials, which is driven by freeenergy minimization $(13,16)$. The solution of DTT7Me in toluene experiences interplay of the energetic parameters at the solvent/copper interfaces under the template bars and solvent/air interfaces inside the holes (see Figure 1) at the upper side. On the contrary, at the bottom-side, the energetic interplay is driven by the surface energy of the device substrate. 

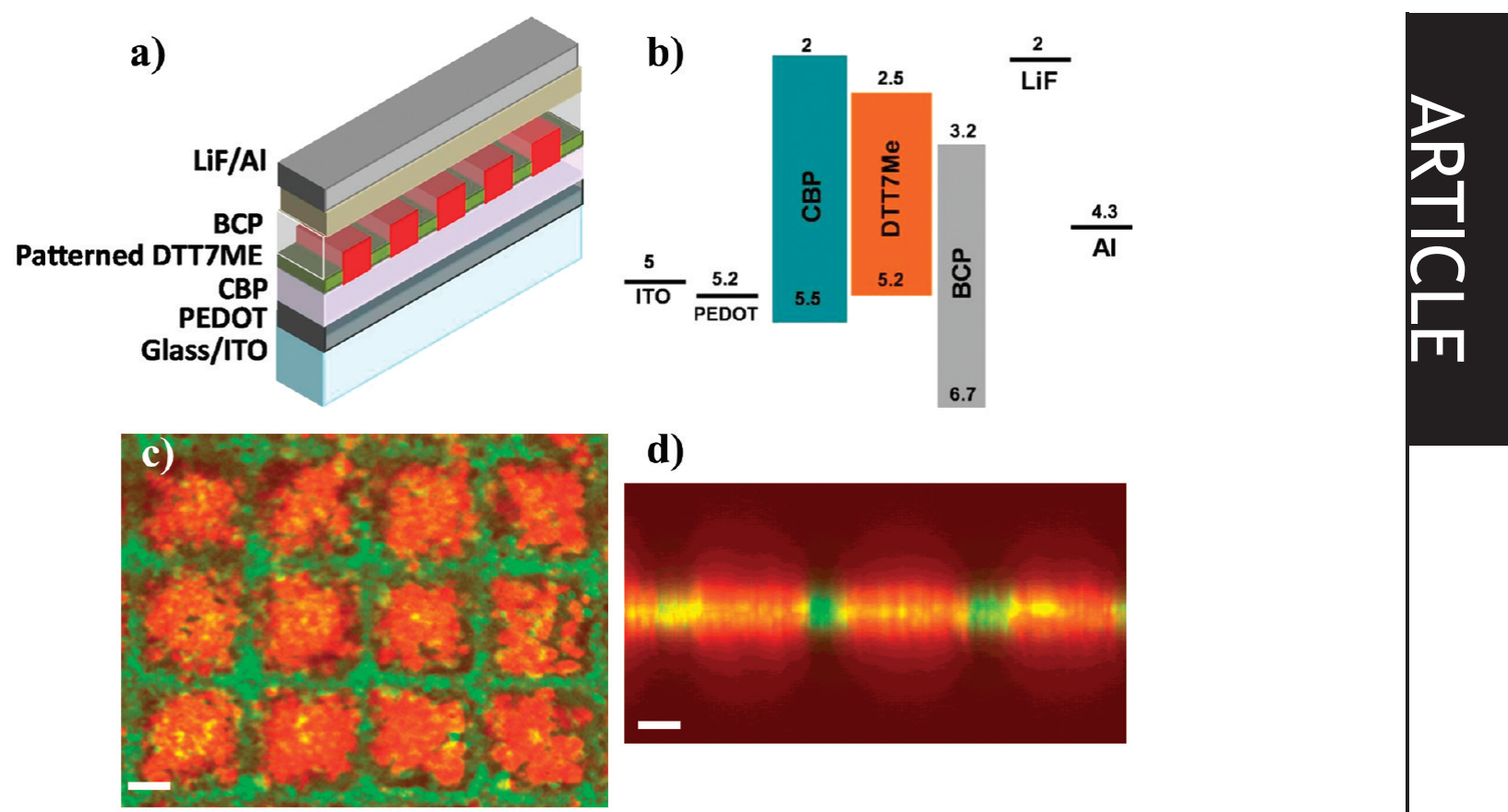

d)

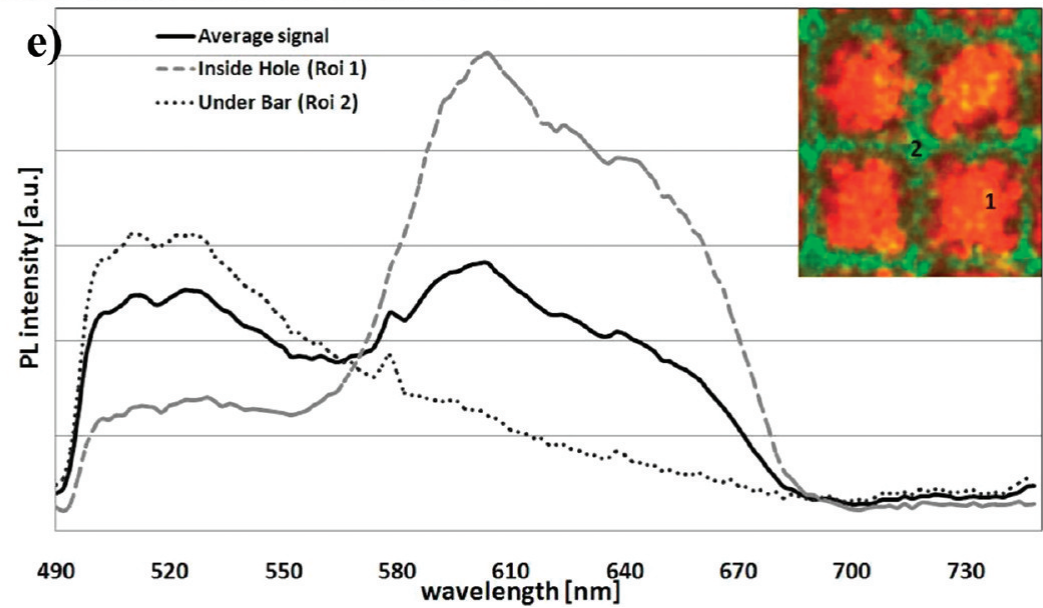

FIGURE 4. (a) Structure of the device ITO// PEDOT:PSS//CBP//DTT7ME//BCP//LiF/Al and (b) the simplified energy level diagram. (c) Multichannels laser scanning confocal microscopy image of the DTT7Me layer of OLED patterned by STD technique; (d) 3D reconstruction of the spatial resolved PL emission, obtained by $x-z$ optical sections of confocal scans; (e) PL spectra collected by confocal microscopy in zones inside (dashed line) and under (dotted line) the grid mesh. The scale bars in $\mathrm{c}$ and $\mathrm{d}$ correspond to $10 \mu \mathrm{m}$.

In our experiment, both PEDOT-PSS and CBP layers have been used as substrates. For the deposition of the organic material both STD technique and drop-cast have been investigated and compared in term of supramolecular organization of the compound. STD process induces meaningful differences in the supra-molecular packing of DTT7Me on PEDOT-PSS (sample A) and on CBP substrates (sample B). On the contrary, drop cast deposition of DTT7Me, realized as reference process, is comparable on both substrates (samples C, D) showing a mix of amorphous and crystalline phases (1 $1 \mathrm{~b})$.

The modulation of molecular arrangement in the solidstate, driven by the STD process, is probably due to the difference in the polar and dispersion contributes to the surface energy $(\gamma)$, as reported in Table 1. Surface energy at the different interfaces attests the possibility to modulate the dynamics of STD lithography by acting on the balance of van der Waals interactions as well as on the control of wetting and dewetting properties during the solvent evaporation. In fact, we observe an alternation of $\gamma$ between the confined copper $(\mathrm{Cu})$ interface and the open interfaces, respectively of PEDOT-PSS and CBP. More importantly, for the open interfaces, we find a higher dispersion contribute of $\gamma$ for the CBP interface, attesting a better affinity with the intrinsically hydrophobic thiophene-based materials.

So far as we have observed for the bicolor STD patterns, the dithienothiophene deposited on CBP substrate (sample B) showed a better conformational morphology of the pixelated structure with respect to that on PEDOT-PSS (sample A) in term of regular organization and spatial periodic alternation of crystalline and amorphous phases across the pattern. Atomic force microscopy (AFM) characterizations attest that well-defined plate crystals are formed inside the mesh squares, with a more oval shape on the PEDOT-PSS substrate (Figure $2 \mathrm{a}-\mathrm{c}$ ) and hexagonal shape on the CBP substrate (Figure $2 d-f$ ). The hexagonal shape is comparable 

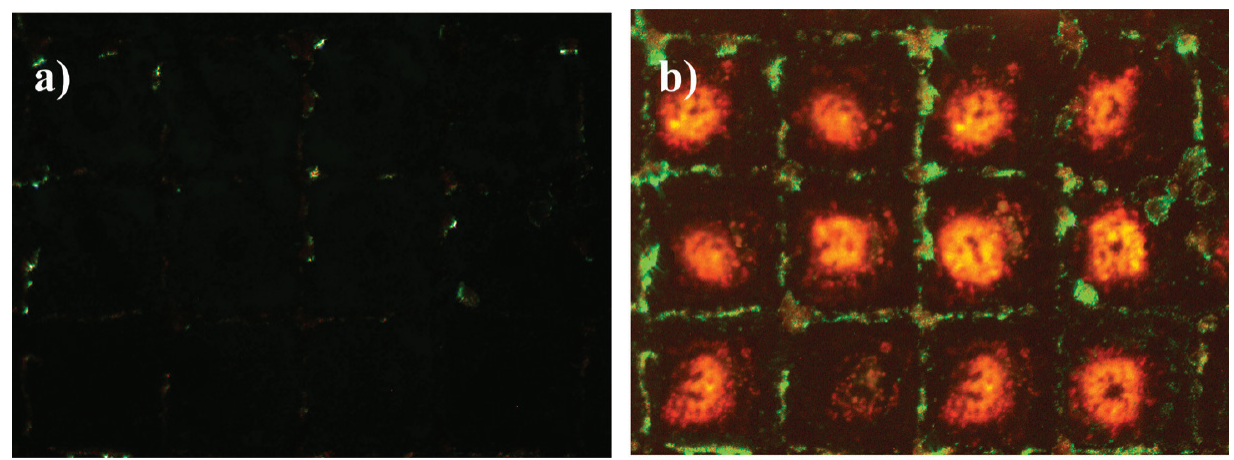

FIGURE 5. (a) Optical image in transmission from the bicolor pixel microarray OLED in the OFF state and (b) optical image, in true colors, of the electroluminescence from the device switched $\mathrm{ON}$ at a bias of $10 \mathrm{~V}$.

with the spontaneous self-assembly of molecular material in planar conformation (11b). The amorphous phase, present under the template bars, is more defined and periodically arranged on the CBP layer (Figure $2 \mathrm{~g}-\mathrm{i}$ ). The peculiar morphology of the supra-molecular organization of sample B can be attributed to the lower surface energy and the stronger dispersion contribute of CBP (see Table 1), which allows a better alternation between the amorphous phase and crystalline conformations. As a consequence, we observed the formation of two regularly alternating regions with well-defined micrometer features and emission at two different wavelengths.

Taking these differences into account, we proceed by investigating the process kinetics. We found out that the differences in supramolecular packing due to the selforganization occuring during the nucleation phenomena can be enhanced and selectively addressed by controlling the process parameters.

We varied the system temperature during the STD process to deeper understand the dynamical evolution of the dewetting process. Slow and fast processes, respectively at $T_{\text {slow }}=24^{\circ} \mathrm{C}$ and $T_{\text {fast }}=80^{\circ} \mathrm{C}$, have been carried out both on PEDOT-PSS and on CBP substrates (samples A, B). The morphology of the different samples has been compared in order to understand the effects of the temperature. In particular, we observed that, during the confined STD process, thermodynamics influences especially the amorphous phase that seems to form prevalently under "fast evaporation" conditions. Figure 3 shows the slow and fast STD deposition of DTT7Me on CBP substrate and underlines differences in the supramolecular packing of amorphous and crystalline phases. The corresponding characterizations for fast and slow deposition on PEDOT-PSS are reported in the Supporting Information.

These results can be explained by considering that slow kinetics makes easier the molecules to organize in a crystalline packing with planar single molecule conformation, which corresponds to the more energetically convenient arrangement (see Figure 3a) (1 1 b).

On the contrary, during the fast process $\left(T_{\text {fast }}\right)$, the liquid instabilities at the solution interface are strongly enhanced and the reduction of the evaporation time contributes to freeze some degrees of freedom, thus allowing the coexist- ence of both the planar crystalline configuration and of the more distorted amorphous phase (see Figure $3 b$ ).

After being able to control the formation of well-defined bicolor regions we explore the realization of a pixelated bicolor OLED. To this aim, we realized a series of devices by STD deposition of DTT7Me both on ITO/PEDOT:PSS (A) and ITO/PEDOT:PSS/CBP interfaces (B) (see Figure 4a,b). $\mathrm{BCP}$ and LiF/Al layers acting as hole blocking layer and cathode, respectively, have been deposited to complete the device.

As reference device, we fabricated OLED with the same multilayer structure, but with DTT7Me deposited by dropcasting without the geometric confinement (device figure not shown). For both of the deposition procedures, STD and drop-cast, we have performed the control of the solvent evaporation rate and we have realized different devices by performing both slow $\left(T_{\text {slow }}\right)$ and fast dewetting process ( $T_{\text {fast }}$.

Integrated and spatially resolved photoluminescence (PL) spectra, collected by a confocal microscope on the pixelated DTT7Me OLEDs, allowed to investigate the optical properties of the STD patterned active layer directly from device. Figure $4 \mathrm{c}-\mathrm{e}$ shows the confocal characterizations of the light emitting devices realized on the CBP layer (B). To attest the effective molecular spatial arrangement and the relative optical properties of the DTT7Me active layer, we have recorded optical spectra under the OLED metallic cathodes by varying the focus depth (Figure 4c). Confocal $x-z$ optical section in Figure $4 \mathrm{~d}$ renders a 3D vision of the organic active layer inside the device and shows regular alternating redand green-light emitting regions, with micrometer-size, inside the device. The green and the red emission bands of the PL emission, in Figure 4e, can be individually attributed to different regions of the patterned film and correspond, respectively, to the region under the template bars and inside the mesh square. The same figures, not shown here, are for the device on PEDOT-PSS.

All these results attest that the OLED fabrication steps, following the STD lithographic process, do not affect the conformational and optical properties of the thiophenebased molecular material.

The final bicolor pixelated organic device, realized on CBP interface and under fast dewetting conditions, is shown in 

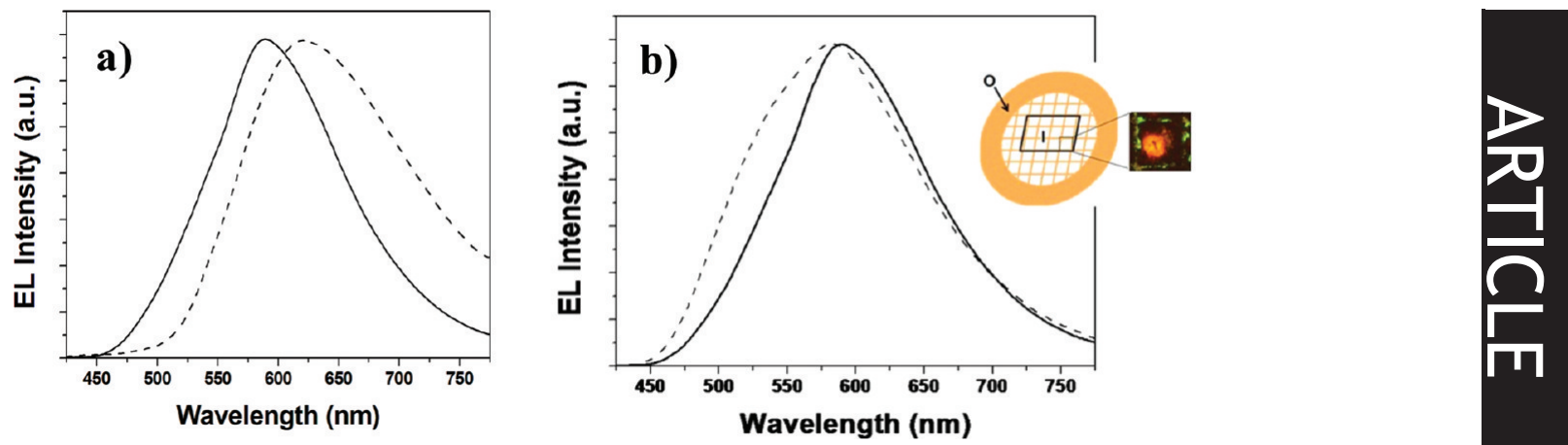

FIGURE 6. EL spectra of DTT7Me OLED deposited by (a) drop casting (solid line fast and dashed line slow) and (b) STD technique on ITO/ PEDOT:PSS/CBP substrate (solid line) zone O outside the grid, (dashed line) zone I inside the grid. The STD bicolor pixel array OLED has been switched on at a bias of $10 \mathrm{~V}$ and the optical image of the device ON is reported in the inset.

Figure 5 which reports the DTT7Me OLED in the OFF (Figure $5 a)$ and $\mathrm{ON}$ (Figure $5 \mathrm{~b}$ ) state. The electroluminescence (EL) from the patterned area (dashed line Figure 6b) clearly shows both the green- and the red-light emissions. The spectrum of the patterned area is characterized by a main emission peak around $600 \mathrm{~nm}$ and an additional hump around $545 \mathrm{~nm}$, which are respectively due to the red emission from the holes and the green emission coming from the well-defined bars (as seen in Figure 4c). Such emissions correspond to the same emission active areas observed in the PL spectra collected by the spatially resolved confocal from the DTT7Me layer inside the device (see Figure $4 \mathrm{e})$. The bicolor emission is not completely defined in the zone outside the grid (solid line Figure 6b) where the supramolecular arrangement is similar to the corresponding drop-cast sample realized in the same conditions (solid line Figure 6a). The luminance recorded at $10 \mathrm{~V}$ is $75 \mathrm{~cd} / \mathrm{m}^{2}$ (see the Supporting Information). By optimizing the thicknesses of the patterned materials and of the others layer, it will be possible to further increase the performances without compromising the bicolor emission.

To attest the effect of the molecular kinetics on the emission properties, we have collected the EL emission of all samples realized by using both slow and fast deposition processes (see the Supporting Information).

\section{CONCLUSIONS}

We have demonstrated the fabrication of the first bicolor micropixelated OLED from a single molecular material by using a single-step procedure. The implementation of a deposition technique, based on a spatial-switch and conformational-sensitive surface-tension-driven lithography, has allowed us to exploit the spontaneous supramolecular properties and the conformational flexibility of a conjugated thiophene-based material, DTT7Me. We proved that we were able to control the STD technique. By optimizing the deposition procedure and by using fast kinetic processes we were able to preserve the optical properties of the active layer in the device structure. This demonstrates that it is possible to control the formation of two regularly alternating emitting regions on a micrometer scale and to obtain electroluminescent emission at two different wavelengths from a single material. By further optimizing the procedure and the device structure, we believe that this technique can be considered a potential tool for the fabrication of real devices by a single-step lithographic process.

Acknowledgment. The authors gratefully acknowledge S. D'Amone for technical support. This work has been partially funded by MIUR (FIRB RBNE03S7XZ_005 "SYNERGY").

Supporting Information Available: Optical microscope images, electroluminescence spectra, and description of experimental procedures (PDF). This material is available free of charge via the Internet at http://pubs.acs.org.

\section{REFERENCES AND NOTES}

(1) (a) Tang, C. W.; Van Slyke, S. A. Appl. Phys. Lett. 1987, 51, $913-$ 915. (b) Friend, R.; Gymer, R. W.; Holmes, A. B.; Burroughes, J. H.; Marks, R. N.; Taliani, C.; Bradley, D. D. C.; Dos Santos, D. A.; Brédas, J. L.; Lögdlund, M.; Salaneck, W. R. Nature 1999, 397, 121-128. (c) Sheats, J. R. J. Mater. Res. 2004, 19, 1974-1989.

(2) (a) Muller, C. D.; Falcou, A.; Reckefuss, N.; Rojahn, M.; Wiederhirn, V.; Rudati, P.; Frohne, H.; Nuyken, O.; Becker, H.; Meerholz, K. Nature 2003, 421, 829-833. (b) Gather, M. C.; Köhnen, A.; Falcou, A.; Becker, H.; Meerholz, K. Adv. Funct. Mater. 2007, 17, 191-200.

(3) (a) Rogers, J. A.; Paul, K. E.; Jackman, R. J.; Whitesides, G. M. Appl. Phys. Lett. 1997, 70, 2658-2660. (b) Lee, T. W.; Zaumseil, J.; Bao, Z.; Hsu, J. W. P.; Rogers, J. A. Proc. Natl. Acad. Sci. U.S.A. 2004, 101 (2), 429-433. (c) Lee, T. W.; Jeon, S.; Maria, J.; Zaumseil, J.; Bao, Z.; Hsu, J. W. P.; Rogers, J. A. Adv. Funct. Mater. 2005, 15, $1435-1439$.

(4) (a) Menard, E.; Meitl, M. A.; Sun, Y.; Park, J. U.; Shir, D. J. L.; Nam, J. S.; Jeon, S.; Rogers, J. A. Chem. Rev. 2007, 107, 1117-1160. (b) Choi, J.; Kim, D.; Yoo, P. J.; Lee, H. H. Adv. Mater. 2005, 17, 166 170.

(5) Cavallini, M.; Albonetti, C.; Biscarini, F. Adv. Mater. 2009, 21, 1043-1053.

(6) (a) Surin, M.; Cho, S.; Yuen, J. D.; Wang, G.; Lee, K.; Leclère, Ph.; Lazzaroni, R.; Moses, D.; Heeger, A. J. J. Appl. Phys. 2006, 100, 033712. (b) Leclère, Ph.; Surin, M.; Bracorens, P.; Cavallini, M.; Biscarini, F.; Lazzaroni, R. Mater. Sci. Eng., R 2006, 55, 1-56.

(7) (a) Gigli, G.; Barbarella, G.; Favaretto, L.; Cacialli, F.; Cingolani, R. Appl. Phys. Lett. 1999, 75, 439-441. (b) Gigli, G.; Della Sala, F.; Lomascolo, M.; Anni, M.; Barbarella, G.; Di Carlo, A.; Lugli, P.; Cingolani, R. Phys. Rev. Lett. 2001, 86, 167-170.

(8) (a) Gigli, G.; Anni, M.; Cingolani, R.; Barbarella, G. Advanced Semiconductor and Organic Nano-Techniques; Morkoc, H., Ed.; Elsevier, New York, 2003; Chapter 5, pp 241-291. (b) Barbarella, G.; Melucci, M.; Sotgiu, G. Adv. Mater. 2005, 17, 1581-1593.

(9) (a) Burroughes, J. H.; Bradley, D. D. C.; Brown, A. R.; Marks, R. N.; Mackay, K.; Friend, R. H.; Burns, P. L.; Holmes, A. B. Nature 1990, 347, 539-541. (b) Hoeben, F. J. M.; Jonkheijm, P.; Mejier, E. W.; Schenning, A. P. H. J. Chem. Rev. 2005, 105, 1491-1546. 


\section{M.; Antolini, L.; Della Sala, F.; Di Carlo, A.; Lugli, P. Appl. Phys.}

(10) Gigli, G.; Lomascolo, M.; Cingolani, R.; Barbarella, G.; Zambianchi, Lett. 1998, 73, 2414-2416.

L (11) (a) Viola, I.; Mazzeo, M.; Passabi, A.; D’Amone, S.; Cingolani, R.; Gigli, G. Adv. Mater. 2005, 17, 2935-2939. (b) Viola, I.; Della Sala, F.; Piacenza, M.; Favaretto, L.; Gazzano, M.; Anni, M.; Barbarella, G.; Cingolani, R.; Gigli, G. Adv. Mater. 2007, 19, 1597-1602.

(12) Cicoira, F.; Santato, C.; Melucci, M.; Favaretto, L.; Gazzano, M. Muccini, M.; Barbarella, G. Adv. Mater. 2006, 18, 169-174.

(13) (a) Karthaus, O.; Koito, T.; Shimomura, M. Mater. Sci. Eng., C 1999, 8-9, 523-526. (b) Harkema, S.; Schäffer, E.; Morariu, M. D.; Steiner, U. Langmuir 2003, 19, 9714-9718. (c) Maillard, M.; Motte, L.; Pileni, M. P. Adv. Mater. 2001, 13, 200-204.
(14) Deegan, R. D.; Bakajin, O.; Dupont, T. F.; Huber, G.; Nagel, S. R.; Witten, T. A. Nature 1997, 389, 827-829.

(15) (a) Piacenza, M.; Della Sala, F.; Fabiano, E.; Maiolo, T.; Gigli, G. J. Comput. Chem. 2008, 29, 451-457. (b) Melucci, M.; Favaretto, L.; Bettini, C.; Gazzano, M.; Camaioni, N.; Maccagnani, P.; Ostoja, P.; Monari, M.; Barbarella, G. Chem.-Eur.J. 2007, 13, 10046 10054.

(16) (a) Israelachvili, J. Intermolecular and Surface Forces; Academic Press: London, 1985. (b) Myers, D. Surface, Interface, and Colloids; VCH: New York, 1991.

AM9007227 